\title{
Prehypertension—new insights for health risks
}

Tianyu Xu and Yuli Huang

We read the Review by Egan and StevensFabry (Prehypertension-prevalence, health risks, and management strategies. Nat. Rev. Cardiol. 12, 289-300; 2015) ${ }^{1}$ with great interest. We wish to discuss some new insights for health risks and management in prehypertension.

First, that prehypertension is associated with increased risks of coronary heart disease (CHD), stroke, and total cardiovascular disease was well reviewed by Egan and Stevens-Fabry. ${ }^{1}$ However, the risks of damage to target organs induced by abnormal blood pressure vary significantly between different ethnic groups. For example, in clinical trials of antihypertensive therapy, the ratio of stroke:myocardial infarction has been reported as being between 5:1 and 8:1 in Chinese individuals and 1:1 in North American and European individuals (traditionally defined as 'Westerners'). ${ }^{2}$ Comprising data for 591,664 individuals, our meta-analysis published earlier this year showed that the increased CHD risk associated with higher-than-optimal blood pressure (that is, $>120 / 80 \mathrm{mmHg}$ ) is greater among Westerners than Asian patients (70\% versus $25 \%$ greater CHD risk, respectively). ${ }^{3}$ Population-attributable risk calculations indicated that $8.4 \%$ of CHD in Asian individuals was attributed to prehypertension, whereas this proportion was $24.1 \%$ in Westerners. ${ }^{3}$ Therefore, the association between prehypertension and CHD risk seems to be stronger for Westerners than for Asians. ${ }^{4}$ These findings support the heterogeneity of targetorgan damage caused by prehypertension among different ethnicities and underscore the particular importance of prevention of CHD in Westerners with prehypertension. ${ }^{3}$
Second, chronic kidney disease (CKD) is another common morbidity of hypertension. Although cross-sectional studies have also shown that prehypertension is associated with $\mathrm{CKD},{ }^{5,6}$ using these studies to establish the detrimental effects of prehypertension on the kidneys is difficult, because kidney diseases per se can elevate blood pressure. However, evidence for a link between prehypertension and risk of CKD is growing stronger. ${ }^{7}$ In our meta-analysis of data from $1,003,793$ individuals, after controlling for multiple cardiovascular risk factors, prehypertension was associated with a significantly increased risk of end-stage renal disease (relative risk 1.59, 95\% CI 1.39-1.91). ${ }^{8}$ The results were consistent across age, sex, ethnicity, and trial characteristics. ${ }^{8}$ Of note, although this information is important to health professionals and those engaged in the prevention of CKD, no available evidence shows that treatment targeting prehypertension results in better clinical outcomes among patients with kidney disease. ${ }^{9}$ Given the low incidence of end-stage renal disease, previous studies might not have been adequately powered, or might not have had an adequate follow-up duration, to detect the difference in incidence of end-stage renal disease.

Taken together, studies have shown robust and significant associations between prehypertension and multiple conditions linked with risk of target-organ damage, including stroke, CHD, and CKD. However, targetorgan damage caused by prehypertension might vary between different ethnicities. ${ }^{3}$ We believe future clinical trials are needed to determine whether interventions for prehypertension have different effects on risk of target-organ damage in different ethnicities.
First School of Clinical Medicine, Southern Medical University, 1838 North Guangzhou Avenue, Guangzhou 510515, China (T.X.). Department of Cardiology, Affiliated Hospital at Shunde (First People's Hospital of Shunde), Southern Medical University, Penglai Road 1, Daliang Town, Shunde District, Foshan 528300, China (Y.H.).

Correspondence to: Y.H. hyuli821@163.com

\section{Competing interests}

The authors declare no competing interests.

1. Egan, B. M. \& Stevens-Fabry, S. Prehypertension —prevalence, health risks, and management strategies. Nat. Rev. Cardiol. 12, 289-300 (2015).

2. Liu, L. S. et al. 2010 Chinese guidelines for the management of hypertension [Chinese]. Zhonghua Xin Xue Guan Bing Za Zhi 39, 579-615 (2011).

3. Huang, Y. et al. Prehypertension and the risk of coronary heart disease in Asian and Western populations: a meta-analysis. J. Am. Heart Assoc. 4, e001519 (2015).

4. Habib, G. B., Virani, S. S. \& Jneid, H. Is 2015 the primetime year for prehypertension? Prehypertension: a cardiovascular risk factor or simply a risk marker? J. Am. Heart Assoc. 4, e001792 (2015).

5. Yano, Y. et al. Association between prehypertension and chronic kidney disease in the Japanese general population. Kidney Int. 81, 293-299 (2012).

6. Crews, D. C. et al. Prevalence of chronic kidney disease in persons with undiagnosed or prehypertension in the United States. Hypertension 55, 1102-1109 (2010).

7. Holmes, D. End-stage renal disease: link between prehypertension and risk of ESRD grows stronger. Nat. Rev. Nephrol. 9, 695 (2013).

8. Huang, Y. et al. Prehypertension and incidence of ESRD: a systematic review and metaanalysis. Am. J. Kidney Dis. 63, 76-83 (2014).

9. Upadhyay, A., Earley, A., Haynes, S. M. \& Uhlig, K. Systematic review: blood pressure target in chronic kidney disease and proteinuria as an effect modifier. Ann. Intern. Med. 154, 541-548 (2011). 